\title{
Weld integrity of the superconducting cable aluminium jackets of W7-X
}

Joris Fellinger, Andrzej Dudek, Daniel Zacharias, Harald Dutz, Konrad Risse, Dag Hathiramani, Victor

Bykov, Felix Schauer

Max-Planck-Institut für Plasmaphysik, EURATOM Association, Teilinstitut Greifswald, Wendelsteinstraße 1, D-17491 Greifswald, Germany

\begin{abstract}
The Wendelstein 7-X (W7-X) modular stellarator is under construction at the Max-Planck-Institut für Plasmaphysik in Greifswald, Germany. The W7-X magnet system contains 70 coils made up from helium cooled superconducting cables in aluminium alloy (EN AW-6063) jackets. Several hundred connections of the jacket to the cable joints are made by aluminium-to-aluminium welds.
\end{abstract}

Due to geometrical and thermal boundary conditions these welds cannot be accomplished free from defects. Microscopic analyses of the welds show that a variety of small flaws such as cracks and pores develop during welding. The welds have thus to be dimensioned accordingly, and appropriate weld qualification, investigation and testing has to be done in order not to jeopardise the structural integrity and leak tightness. The weld is mechanically loaded during cool-down due to the difference in thermal contractions between the GRP insulation and the aluminium, and during operation by bending moments from electromagnetic forces. An extensive number of different pressure, bending, and pulling tests were performed over the years in order to verify sufficient quality of the welds.

This paper is concerned with loading of the weld during cool-down in cable axis direction. It is shown that a series of simple pulling tests on the conductor jacket, welded to the cable joint, indicates that the failure starts in the heat affected zone of the jacket rather than at the weld tips where the maximal influence of welding irregularities and the notch effect is expected. In order to investigate the mechanical load distribution in the weld region, a 3D FE model was created for help in judging the criticality of weld flaws. In the course of these investigations a simple load configuration for a fast approval test of the weld was developed that can be used for the routine qualification of the welding process before real welding in W7-X takes place. Currently, acceptance criteria for the test are under development. 


\section{Introduction}

The magnet system of the Wendelstein 7-X (W7-X) modular stellarator has fivefold symmetry and incorporates five types of non-planar coils and two types of planar coils, attached to the support structure. The winding pack of the coils and the bus system are produced from cable-in-conduit conductors (CICCs) whose jackets are extruded from aluminium EN AW 6063 [1]. The conductors of each coil are mutually interconnected, and the outgoing ends are also connected to the conductors of the busbar system by a specially developed busbar joint. In order to avoid an on-site junction between the stainless steel of this joint and the aluminium of the jacket, a friction welded transition part from an external supplier is employed that consists at one side of stainless steel and at the other side of aluminium alloy EN AW 6061 T6 [2]. The aluminium cable jackets are welded to the aluminium side of the transition part, see figure 1. A TIG weld process is applied using an AlSi5 filler of $2 \mathrm{~mm}$ diameter. The welding parameters were optimised at IPP. In addition, the transition part and filler are dried for 24 hours under vacuum conditions at $70^{\circ} \mathrm{C}$ in order to minimise the flaws in the weld.

The welded ends of the jackets are electrically insulated by glass fibre reinforced epoxy (GRP) wrapping. The GRP insulation of the busbar jacket is additionally interleaved with two Kapton foils. Around the weld region, a prefabricated GRP cap is included in the wrapping with the aim of increasing the strength of the connection, see figure 2 .

Obviously, the jackets and all joints must be helium tight during all modes of operation. The leakage rate limit for each welded connection has been set to $10^{-9} \mathrm{mbar} \cdot \mathrm{l} / \mathrm{s}$.

The weld is a critical zone as the welding process introduces, due to given boundary conditions, unavoidable irregularities such as pores and cracks, and the heat input reduces the mechanical properties of the fused zone and especially the heat affected zone (HAZ) of the heat treatable Al-alloy. The stresses that arise within the weld region during cool-down are assessed analytically and confirmed by numerical calculations showing that the weld and the HAZ are highly loaded, not only due to electromagnetic forces but due to cool-down as well.

Obviously, the weld resistance plays a decisive role in the risk of overloading. Therefore, an extensive experimental program was carried out to determine the lower bound of the weld resistance. 
Finally, a fast approval test procedure is being developed using various preliminary test setups and 3D finite element (FE) simulations. From the extensive experimental program it was confirmed that the weakest cross-section with respect to axial pulling is within the HAZ rather than in the weld itself. In order to focus the fast test procedure on the weld itself, where the flaws are expected, another loading configuration has been developed and investigated. This permits routine checks of the welding process before real welding within the W7-X machine.

\section{Criticality of the weld}

The weld is a critical part with respect to high vacuum cold helium tightness. Cable jackets belonging to the coil are artificially aged and thus hardened by heat treatment for 14 hours at $165{ }^{\circ} \mathrm{C}$, and those belonging to the bus system are naturally aged for several months up to several years. The heat input during welding has a detrimental effect on the strength gained during aging of the aluminium alloy.

Microscopic inspections of the weld show that flaws such as cracks and pores develop during welding, especially near the edge of the weld where peak stresses are expected, see figure 3 . Those flaws have a negative effect on the resistance of the weld as they increase peak stresses and could initiate failure cracks. The flaw size varies strongly between the weld specimens. Based on sample testing, acceptance criteria have been developed [3]. Most but certainly not all flaws occur at the interfaces between the weld-jacket and weld-transition parts. Therefore, peak stresses that arise near the tips of the weld are of utmost interest.

The aluminium weld between the jacket and the transition part is loaded by electromagnetic forces as well as the differential thermal contractions between the GRP insulation and the aluminium during cool-down. An analytical model on the basis of Bernouilli's hypothesis of plane cross-sections remaining plane has been created for the system which includes the GRP wrapping, the GRP cap, and the Al-alloy jacket. Based on the nominal values of material properties according to table 1 and the nominal cross-sectional areas according to figure 4a, the nominal thermal stress and the corresponding axial force are calculated. For the extreme case of plane cross-sections (i.e. perfect bond and no shear deformation), figure 4b shows that the highest thermal stresses in the jacket of some $77 \mathrm{MPa}$ arises in the cross-section just outside the zone of the GRP cap, some $65 \mathrm{~mm}$ from the Fe-Al interface or some $15 \mathrm{~mm}$ from the weld tip. 
As shown in the next section, this takes place within the HAZ where the yield limit is lower than for the base material.

Any change in the axial force in the aluminium along the axis results in shear stresses causing shear deformation in the solid material. The effect of shear deformation has been assessed through a comparison of the axial force in the aluminium with the results of a linear elastic FE model that was updated with the material properties of table 1 . In figure 5a, a good agreement is shown between both models. The forces of the 1D model correspond to the stresses of figure $4 \mathrm{~b}$. The lower axial force in the FE model is attributed to the inclusion of shear deformation.

The shear stresses can also induce slip, particularly at the aluminium GRP-interface, since the thermal contraction of the GRP in the hoop direction is smaller than that of the aluminium. As a result, the GRP inner diameter decreases less during cool down than the jacket diameter. Tensile stresses will develop on the interface and contribute to the separation. Slip could in principle also occur at the interfaces between the GRP cap and the GRP wrap, but this is less likely since the cap end face forms some kind of shear key, and the bond between epoxy on epoxy is quite good. In the remainder of this section it is therefore assumed that the GRP wrap and GRP cap remain bonded.

When slip occurs between the GRP and the aluminium, plane cross-sections no longer remain plane, except at the end of the unbonded length at the sections where a mechanical fixation between aluminium and GRP prevents the slip. The unbonded length for each joint, if any, is different. The fixation of the GRP at the end of the transition part coincides approximately with the Fe-Al interface inside the transition part. Slip is also prevented where the jacket is curved, because the bending stiffness of the jacket and the GRP wrap resist sliding along a curved section. The dependence of the axial force on the unbonded length for the case on hand with changing cross-sections has been estimated with a second analytical model. The main results are presented in figure 5b. If the cross-sectional areas over the unbonded length are constant, the axial force in the unbonded situation coincides with that of the bonded situation. For large unbonded lengths, the axial force approaches the axial force of the bonded jacket. Clearly, the thermal stress in the HAZ is lower in the unbonded case than in the extreme case of perfect bond. 
The cap has a very large beneficial effect on the thermal stresses due to the higher thermal contraction and lower Young's modulus of the GRP cap in the axial direction in comparison to the GRP wrapping.

Since W7-X is to be exposed to several dozens of thermal cycles and about thousand electromagnetic load cycles, plastic strains need to be minimised with a view to exclude fatigue failure and possible serration effect. Measurements of the mechanical properties of the aluminium have confirmed that the yield stress HAZ is significantly lower than that of the unaffected zone, see table 2.

Even according to the conservative analytical approximation, the peak thermal stress of some $77 \mathrm{MPa}$, (see figure 4b) remains substantially below the yield limit of the HAZ of $132 \mathrm{MPa}$ at $4 \mathrm{~K}$ and of $108 \mathrm{MPa}$ at $77 \mathrm{~K}$. Notably, the cool down load to $77 \mathrm{~K}$ could be critical as nearly all thermal contraction has developed at $77 \mathrm{~K}$ while the strength of the HAZ increases at further cool down to $4 \mathrm{~K}$. So there is sufficient safety margin to avoid plasticity of the HAZ due to cool down.

Concerning plasticity in the welds, the stochastic lower bound of the mechanical resistance of the HAZ was verified, as shown in section 3.

\section{Experimental assessment of lower bound resistance of the HAZ}

In order to determine the lower bound resistance of the HAZ a series of 40 axial tensile tests have been carried out on jackets welded onto dummy transition parts. The test set up has been developed in a number of trial tests. No GRP was applied in these tests. Half of the specimens were subjected to five thermal cycles with cool-down times of 20 minutes to $77 \mathrm{~K}$. The other half was not submitted to any thermal cycle.

In order to apply the load at the transition part, a bolt was screwed into it. At the other side, the jacket was simply clamped by the test machine using tailor made clamps. The specimens were subjected to axial pulling forces at room temperature. During the test, helium leakage was measured until an axial deformation of $2.9 \mathrm{~mm}$ was reached. Hereafter, the specimens were further pulled up to failure without measuring the helium leakage.

All specimens did not fail at the weld itself but in the jacket some $5 \mathrm{~mm}$ away from the weld tip, see figure 6. The failure load amounted $24.15 \mathrm{kN}$ on average with a coefficient of variation of $5.6 \%$. The 
average load corresponds to a mean axial stress of $168 \mathrm{MPa}$. Apparently, irregularities in the weld did not trigger a failure in any of the specimens.

All specimens were helium tight at the start of the test. Three specimens demonstrated helium leakage before a deformation of $2.9 \mathrm{~mm}$ was achieved, but never before reaching $2.6 \mathrm{~mm}$. In all cases, leakage occurred in the descending branch of the load-displacement diagram, i.e. after the failure load had been reached. No effect of thermal cycling was found.

With a view to confirm the role of the HAZ concerning the failure pattern, the extent of the heat affected zone was assessed through hardness measurements [4]. The hardness gradually increases away from the weld over a length of some $30 \mathrm{~mm}$, see figure 7. It means that the observed failure crack in the tensile tests is located inside the HAZ and coincides with the observed hardness minimum. The mean stress of $168 \mathrm{MPa}$ at failure also agrees quite well with the measurements of the tensile strength of the HAZ as given in table 2.

Further tests are planned using micro-strain gauges to get a detailed understanding of the strength distribution along the HAZ.

\section{Development of fast approval test procedure}

In order to ensure a constant weld quality during assembly of W7-X, a fast approval test procedure is being developed with the help of FEA. At least once for every welding application, trial welds have to be made on a dummy sample and tested destructively to check quasi continuously the welding parameters and the welding capability of the craftsman. Primary goal of the test procedure is to prove in a fast and easy way the weld rather than the HAZ of the jacket. Principle idea is to carry out an axial tension test on the weld because of its simplicity and execution speed.

A $45^{\circ}$ and a $180^{\circ}$ 3D FE model as shown in figure $8 \mathrm{a}$, was created to determine the peak stress distributions over the weld when the jacket is subjected to bending moment or axial force. A part of the mesh of the jacket is not shown in this figure while the output paths IN and OUT along the weld tips are plotted in the model. Bending moment causes peak stresses both along path IN and OUT, see figure 8b. Welding irregularities near both paths could therefore be critical for the integrity of the weld. 
On the contrary, a simple axial pulling load at the end of the jacket causes predominantly peak stresses along path OUT, see figure 9. Thus, a test based on such a load configuration could overlook irregularities at the inner weld tip, which could still be critical under bending loads. However, as shown above, a simple pulling test is not relevant for the weld anyway and therefore methods were developed to check the weld proper by pushing the jacket out of the transition part or pulling it from inside via a threaded bolt. These loading configurations were analysed using the $45^{\circ}$ model by adding a central coaxial bolt through which the load was applied and the results of this model are shown in figure 9. The thread of the bolt was modelled with an interface layer of $0.1 \mathrm{~mm}$ thickness. The Young's modulus of the interface layer was varied between 10 and $100 \%$ relative to the Young's modulus of the bolt without significant influence.

With a pushing load on the jacket from the transition part side, path IN is mostly loaded, see figure 9. When the load is applied through the bolt, both paths are loaded in a balanced way.

The effect of the penetration depth of the bolt on the peak stress distribution along path IN and OUT was also analysed. Fortunately, within a range of $20 \mathrm{~mm}$ variation, no significant effect was found on the peak stresses distributions of path IN or OUT. So, in the test, the penetration depth is not a critical parameter for a balanced loading of both paths.

This bolted load configuration was applied in practise. In four tests so far, the failures occurred as intended at the weld rather than through the HAZ, see figure 10a. The failure load was some 1.5 times higher than for the failure of the HAZ in direct pulling tests without bolts.

\section{Conclusions}

The welds between the aluminium alloy jacket of the superconductor and the aluminium alloy transition part need to remain helium tight throughout all operation conditions where they are highly loaded due to cool-down and electro magnetic forces. During cool-down, the differential thermal contraction between the aluminium and the GRP insulation causes thermal stresses in the weld and particularly in the HAZ of the jacket. The GRP cap reduces the thermal stresses significantly due to its higher thermal contraction in axial direction and its lower Young's modulus than the GRP wrapping. The thermal stress remains 
substantially below the yield limit of the HAZ. The risk of plastic fatigue failure over multiple cool down cycles is thus controlled.

Welding irregularities such as pores and cracks increase the sensitivity of the weld to peak stresses and reduce the resistance of the weld. However, in all the tests failures occurred in the HAZ of the jacket at a mean stress level that corresponds to the tensile strength of the HAZ. So, these weld flaws did not trigger any failure in 40 tests.

It has to be continuously assured that weld irregularities do not critically decrease the weld resistance, therefore, the welding process at the machine will be routinely controlled. In order to complement and speed up the currently employed tests based on micrograph investigation, a simple load configuration on welded specimens is being developed in which the pulling load is applied through a bolt screwed into the end of the jacket up to the level of the weld. In this way, the weld is critically assessed as peak stresses develop at both weld tips of the weld-jacket interface without affecting the generally weaker HAZ. Next step in the development of the approval test is the derivation of proper acceptance criteria, for which further experimental and numerical investigations are ongoing.

\section{References}

1 Measured by FZK, Karlsruhe, Germany

2 EN 573-1: 2004, Aluminium and Aluminium Alloys - Chemical Composition and Form of Wrought Products - Part 1: Numerical Designation System, CEN Brussels, Belgium, 2004

3 M. Schröder, J. Holluba, K. Heyn, Quality assurance of aluminium weld seams and cast casings of the W7-X coils, this conference

4 G. Winkel, Härteprüfung an Al-Schweißnähten / Verbindung Hülse-Supraleiter, 2007, SLV ref. PB210-070112-01 


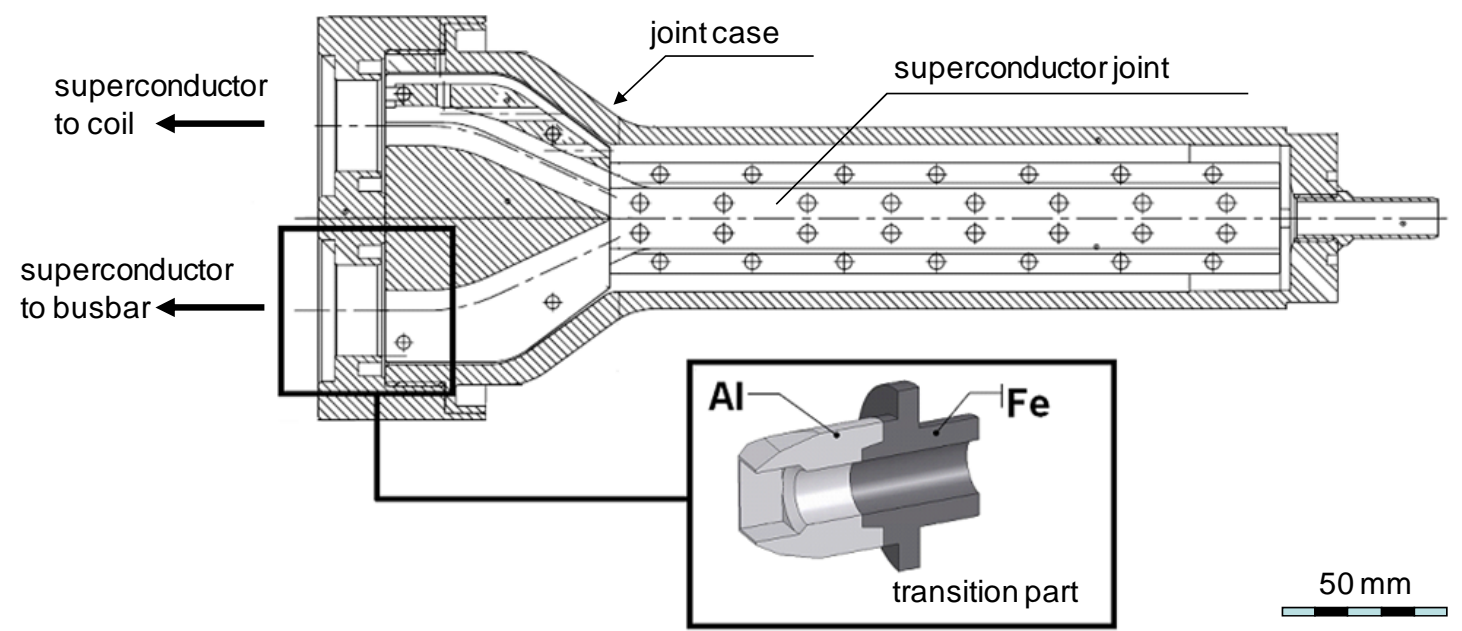

Figure 1: Joint with transition parts to connect the superconductors.

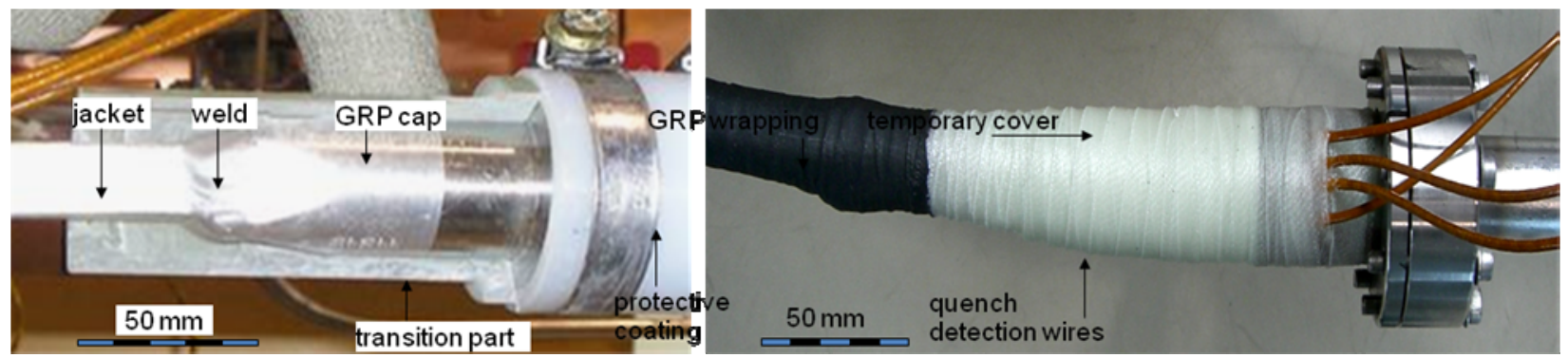

Figure 2: Photo during (left) and after (right) wrapping of the GRP around the welded connection. One half of the prefabricated GRP cap is already in place in the left picture. The wires in the right figure protruding from the insulation are for quench detection.

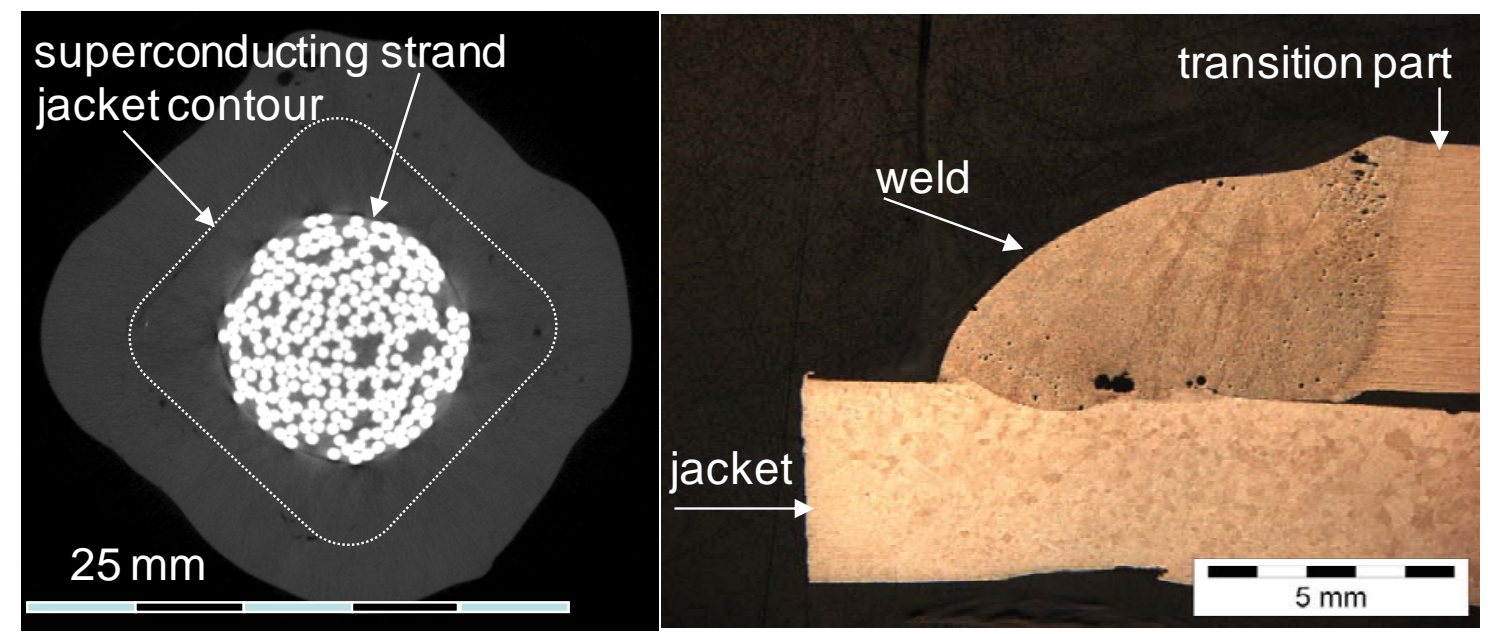

Figure 3: Typical examples of pores and cracks in the weld as observed in a computer tomography scan in axial direction (left) and on a cut section through the weld after etching (right). 

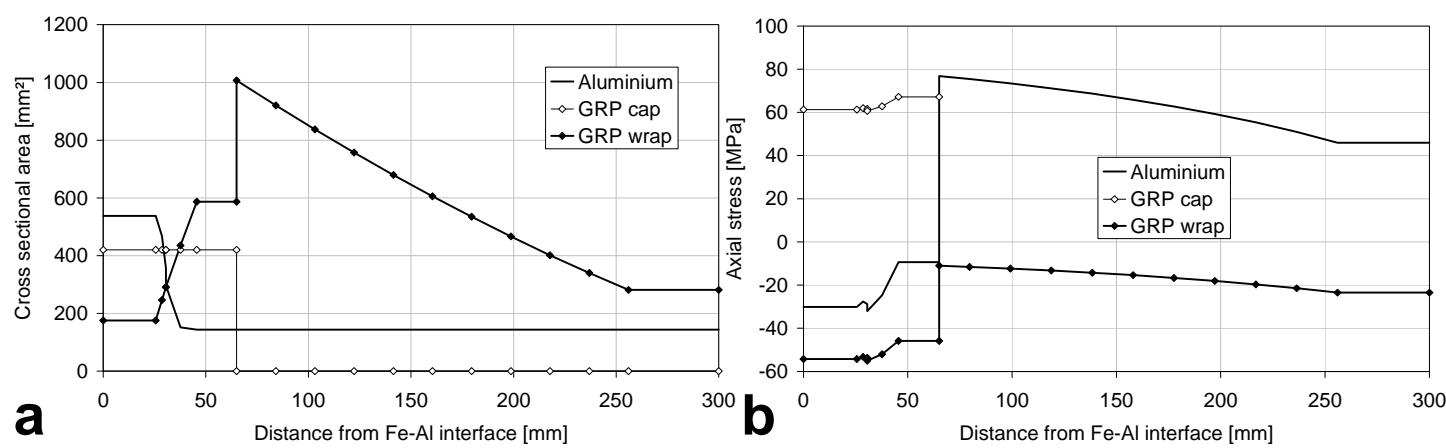

Figure 4: Change along the axis of the cross-sectional area (a) and of the axial stress (b) due to thermal contraction from 293 to $4 \mathrm{~K}$.
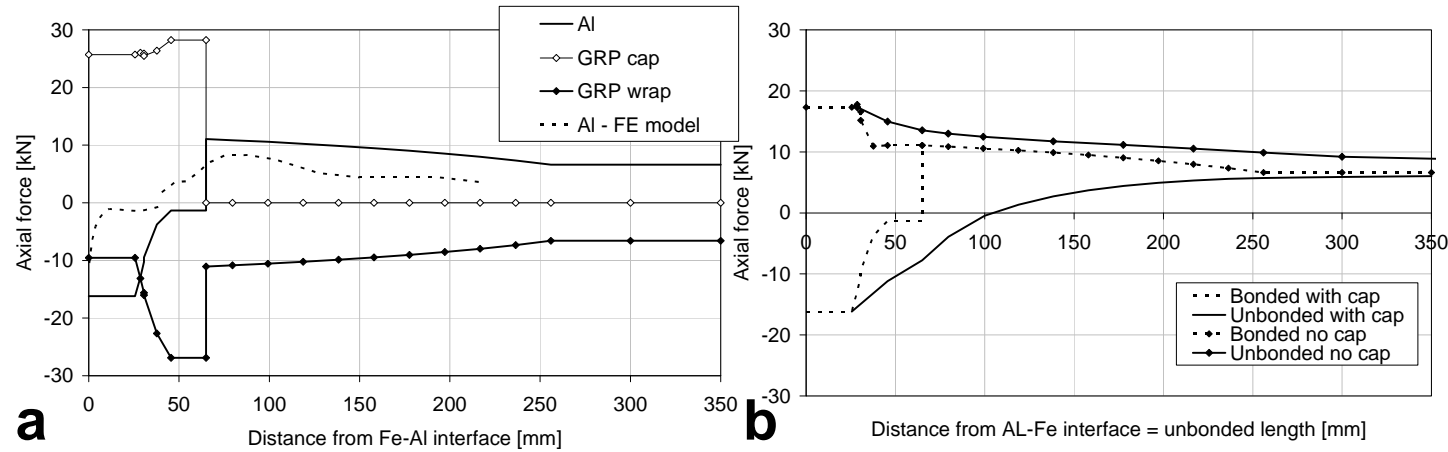

Figure 5: Change along the axis of the axial forces in the bonded situation and comparison with the FE model (a). Axial force in the unbonded situation as function of the unbonded length in comparison with the axial force in the bonded situation for the cases with and without the insulation cap (b).
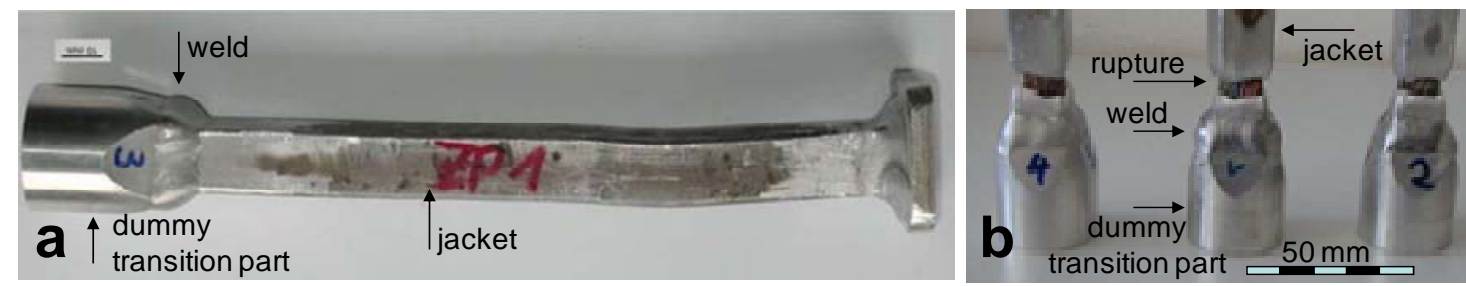

Figure 6: Test sample (a) and typical failure pattern of the axial pulling tests (b).

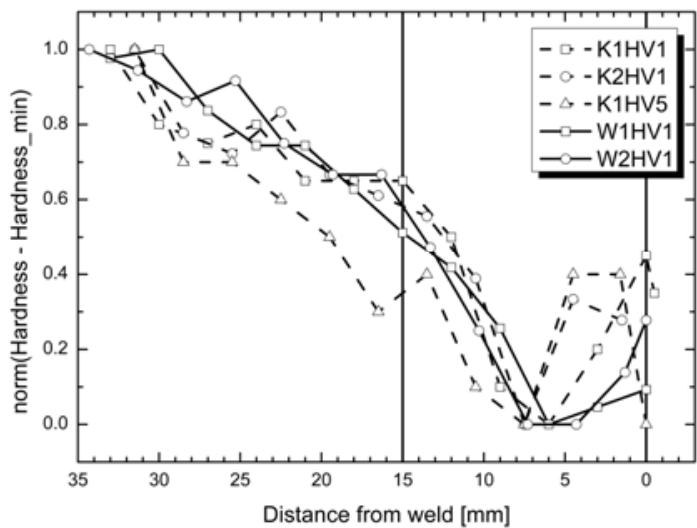

Figure 7: Variation of the Vickers Hardness over the length of the jacket normalised between the highest and smallest values. Heat treated jackets are presented in solid lines, naturally aged jackets in dashed lines. HV1 and HV5 refer to an impact force of $1 \mathrm{~kg}$ and $5 \mathrm{~kg}$, respectively. 

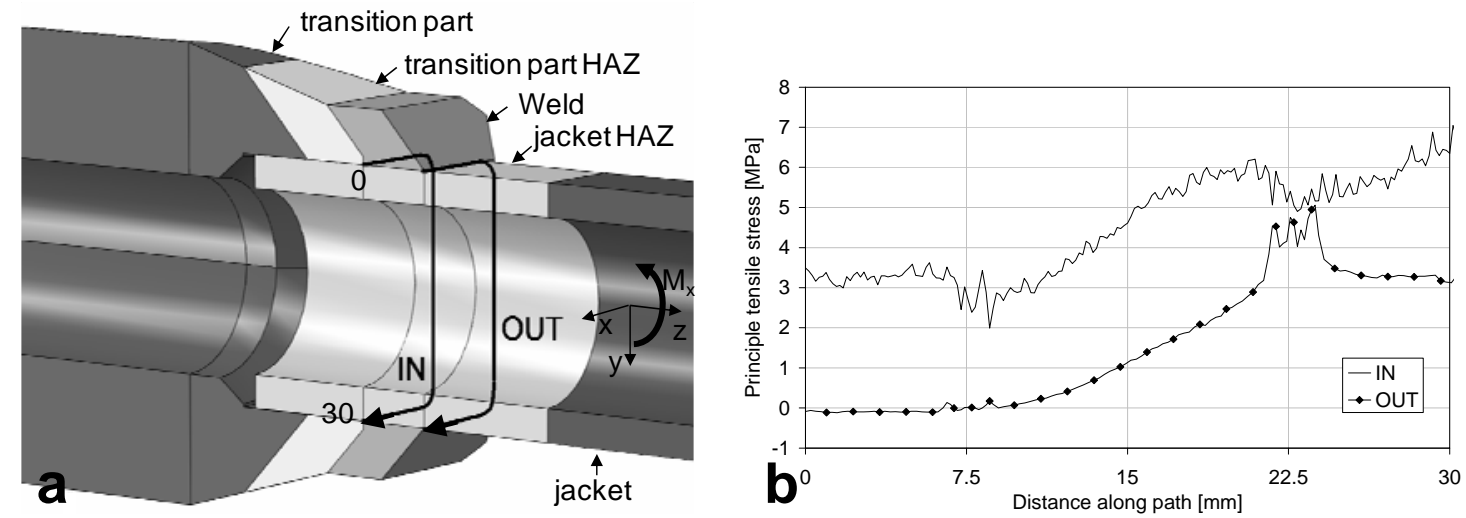

Figure 8: Mesh of the $180^{\circ}$ 3D model with the definition of paths along which the peak stresses were analysed (a). Peak stress distribution along path "IN" and "OUT" due to a bending moment applied at the end of the jacket causing 1 MPa axial stress at the outer fibre of the jacket (b).
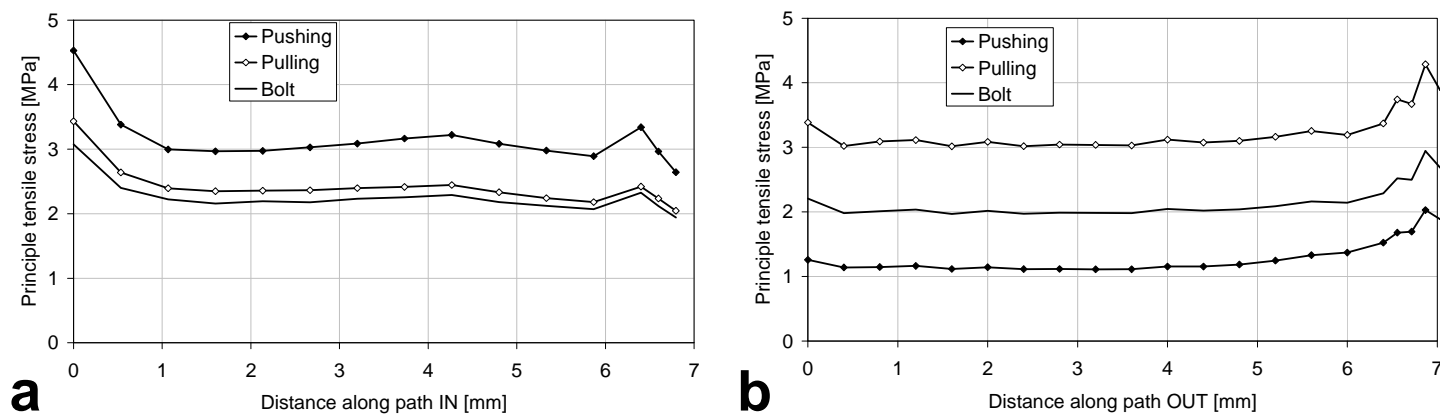

Figure 9: Effect of load configuration on the peak stresses along path IN (a) and OUT (b).
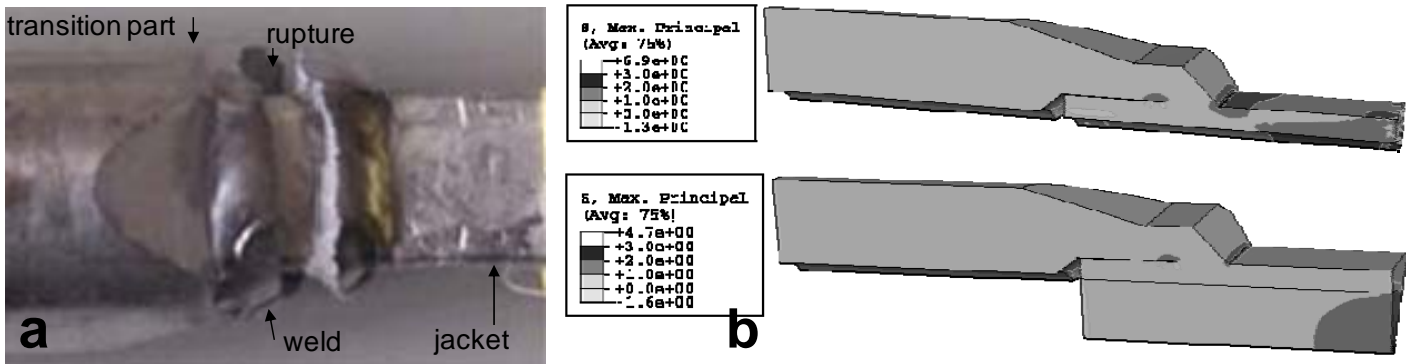

Figure 10: Typical crack pattern through the weld in pulling test using internal bolt (a) and principle tensile stresses in the model with and without the co-axial bolt (b).

Table 1: Mechanical properties of GRP and aluminium as used in jackets

\begin{tabular}{|l|c|c|c|c|c|c|c|c|}
\hline & Temperature & \multicolumn{3}{|c|}{ GRP wrapping [1] } & \multicolumn{3}{c|}{ GRP cap [1] } & Aluminium [1] \\
\cline { 3 - 9 } & {$[\mathrm{K}]$} & axial & Hoop & radial & axial & Hoop & radial & Isotropic \\
\hline Thermal contraction [\%o] & $293 \rightarrow 4$ & -2.94 & -2.77 & -7.25 & -7.56 & -2.55 & -9.10 & -4.30 \\
\hline Young's modulus [GPa] & 7 & 30.1 & 31.3 & 18.2 & 21.5 & 41.8 & 25.5 & 74.3 \\
\hline
\end{tabular}

Table 2: Strength properties of the aluminium alloy depending on aging and welding [1]

\begin{tabular}{|l|c|c|c|c|c|c|c|c|}
\hline & \multicolumn{4}{|c|}{ Naturally aged } & \multicolumn{3}{c|}{ Artificially aged (heat treated) } \\
\cline { 2 - 9 } & Base material & \multicolumn{3}{|c|}{ Welded material } & Base material & \multicolumn{3}{c|}{ Welded material } \\
\hline Temperature [K] & 293 & 293 & 77 & 7 & 293 & 293 & 77 & 7 \\
\hline Yield limit [MPa] & 139 & 97 & 108 & 132 & 231 & 83 & 110 & 134 \\
\hline Tensile strength [MPa] & 228 & 182 & 266 & 286 & 274 & 159 & 245 & 295 \\
\hline
\end{tabular}


